REVIEW ARTICLE OPEN

Check for updates

\title{
The potential use of digital health technologies in the African context: a systematic review of evidence from Ethiopia
}

\author{
Tsegahun Manyazewal $\mathbb{1}^{1 凶}$, Yimtubezinash Woldeamanuel ${ }^{1}$, Henry M. Blumberg ${ }^{2}$, Abebaw Fekadu ${ }^{1}$ and Vincent C. Marconi ${ }^{2}$
}

The World Health Organization (WHO) recently put forth a Global Strategy on Digital Health 2020-2025 with several countries having already achieved key milestones. We aimed to understand whether and how digital health technologies (DHTs) are absorbed in Africa, tracking Ethiopia as a key node. We conducted a systematic review, searching PubMed-MEDLINE, Embase, ScienceDirect, African Journals Online, Cochrane Central Registry of Controlled Trials, ClinicalTrials.gov, and the WHO International Clinical Trials Registry Platform databases from inception to 02 February 2021 for studies of any design that investigated the potential of DHTs in clinical or public health practices in Ethiopia. This review was registered with PROSPERO (CRD42021240645) and it was designed to inform our ongoing DHT-enabled randomized controlled trial (RCT) (ClinicalTrials.gov ID: NCT04216420). We found 27,493 potentially relevant citations, among which 52 studies met the inclusion criteria, comprising a total of 596,128 patients, healthy individuals, and healthcare professionals. The studies involved six DHTs: mHealth (29 studies, 574,649 participants); electronic health records (13 studies, 4534 participants); telemedicine (4 studies, 465 participants); cloud-based application ( 2 studies, 2382 participants); information communication technology (3 studies, 681 participants), and artificial intelligence (1 study, 13,417 participants). The studies targeted six health conditions: maternal and child health (15), infectious diseases (14), non-communicable diseases (3), dermatitis (1), surgery (4), and general health conditions (15). The outcomes of interest were feasibility, usability, willingness or readiness, effectiveness, quality improvement, and knowledge or attitude toward DHTs. Five studies involved RCTs. The analysis showed that although DHTs are a relatively recent phenomenon in Ethiopia, their potential harnessing clinical and public health practices are highly visible. Their adoption and implementation in full capacity require more training, access to better devices such as smartphones, and infrastructure. DHTs hold much promise tackling major clinical and public health backlogs and strengthening the healthcare ecosystem in Ethiopia. More RCTs are needed on emerging DHTs including artificial intelligence, big data, cloud, cybersecurity, telemedicine, and wearable devices to provide robust evidence of their potential use in such settings and to materialize the WHO's Global Strategy on Digital Health.

npj Digital Medicine (2021)4:125; https://doi.org/10.1038/s41746-021-00487-4

\section{INTRODUCTION}

Health technology innovations are transforming the discovery, development, and delivery of health products and services ${ }^{1-4}$ and significantly changing the way health conditions are diagnosed, treated, and prevented ${ }^{5,6}$. These innovations are building a sustainable foundation for affordable, accessible, and high-quality medicines, vaccines, medical devices, and system innovations, pursuing novel solutions, entrepreneurial ventures, and public sector efforts to the most challenging health problems $s^{7,8}$. Digital health technologies (DHTs) ${ }^{9-21}$, pharmacoginomics 22,23 , and process innovations ${ }^{24-27}$ are rapidly emerging as promising health interventions. More innovations are expected to emerge as healthcare demand and spending rise ${ }^{28,29}$. Nevertheless, many of these breakthroughs have not reached the healthcare providers and the people most in need to tackle the rising burden of diseases ${ }^{30-32}$. People living in low-income countries, such as many countries in Africa, are at high risk of many health conditions compared to those living in other regions, while having the most limited access to health innovations ${ }^{30,33}$. Africa has the greatest healthcare challenges in the world: life expectancy is 60 years, substantially lower than the global average of 72 years; maternal mortality ratio is 547 per 100,000 , but 13 in high-income countries and 216 globally; under 5 mortality is 76 per 1000, but 5 in highincome countries and 39 globally $^{34,35}$. While there were 1098 researchers per million inhabitants globally, the corresponding figure for Africa was 87.9 per million ${ }^{35}$. Africa lags in the capacities for health technology innovations, while it bears $23 \%$ of the global disease burden and $16 \%$ of the world population, with the continent expected to double its population by 2050, from 1 billion to nearly 2.4 billion $^{36-40}$.

Without urgent technological, industrial, intellectual, and research-oriented health interventions, Africa cannot tackle the needs and demands of its population. If health technology innovations are needed to transform health system gaps in Africa, it is important to generate country-specific evidence to identify challenges and opportunities in the region as potential resources for further interventions. The World Health Organization (WHO) embraced a more proactive stance in this regard. In 2020, the WHO developed a global strategy on digital health for $2020-2025^{41}$. The vision of the strategy was to improve health for everyone, everywhere by accelerating the development and adoption of appropriate, accessible, affordable, scalable, and sustainable person-centric digital health solutions to prevent, detect and respond to epidemics and pandemics, developing infrastructure and applications that enable countries to use data to promote health and wellbeing, and to achieve the healthrelated United Nations's Sustainable Development Goals (SDGs). Through its Africa office, the WHO Regional Office for Africa

${ }^{1}$ Addis Ababa University, College of Health Sciences, Center for Innovative Drug Development and Therapeutic Trials for Africa (CDT-Africa), Addis Ababa, Ethiopia. ${ }^{2}$ Emory

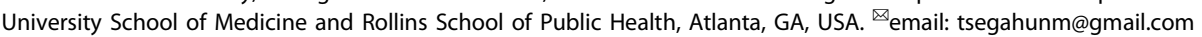


(WHO-AFRO), the WHO designed the health technologies and innovations program to guide the assessments, development, ethics, use, and monitoring of national health technology strategies, with a broader aim of improving access, quality, and rational use of health innovations, including medicines, medical products, and technologies ${ }^{42}$. Similarly, in 2019, the WHO developed a guideline that established recommendations on DHTs for health systems.

This study is in support of the WHO's DHT initiatives. We focused on Ethiopia, the fastest growing economy in Africa per the 2019 World Bank report ${ }^{43}$, and the second most populated country, with more than 117 million people in 2021. Ethiopia aims to reach lower-middle-income status by 2025, with strong commitment and dedication to achieve the SDGs by 2030. The Ethiopian Ministry of Health (MOH) recently, on 06 August 2020, launched a Digital Health Innovation and Learning Center, the first of its kind, where experts can design and validate digital health tools, synthesize and promote best practices, and scale-up innovations ${ }^{44}$. As of 30 April 2021, there were 54.7 million total telecommunication subscribers in the country ${ }^{45}$. Mobile voice subscribers reached 52.8 million, data and internet users 25 million, fixed broadband subscribers 349,000 and fixed service subscribers 923,000 . The telecom population and geographic coverage reached $95 \%$ and $85.4 \%$, respectively and the density reached $50 \%{ }^{45}$. On 22 May 2021, the Ethiopian government awarded a new nationwide telecom license to the Safaricom-led consortium that includes its parent firms Vodafone and Vodacom, British development finance agency CDC Group and Japan's Sumitomo Corporation after submitting a financial bid offering US $\$ 850$ million ${ }^{46}$. The Consortia is expected to invest over $\$ 8$ billion and create jobs for US\$1.5 million citizens. The Ethiopian Health Sector Transformation Plan recognizes the need for improving digital health infrastructure to facilitate equitable access to quality healthcare for all Ethiopians ${ }^{47}$. A systematic review of digital health technology-enabled research in Ethiopia has not been synthesized to inform debates and decisions.

Thus, we aimed to investigate whether and how digital health technologies (DHTs) are absorbed in Africa, tracking Ethiopia as a key node, through a systematic review of available studies.

\section{RESULTS}

\section{Characteristics of included studies}

Study selection: From the 27,493 articles screened, 2397 duplicates were removed and 24,863 were excluded based on the title or abstract. The rest of 233 full-text articles were screened for eligibility, of which 181 were excluded for being irrelevant to the main subject (144) or focused on non-health conditions (37).

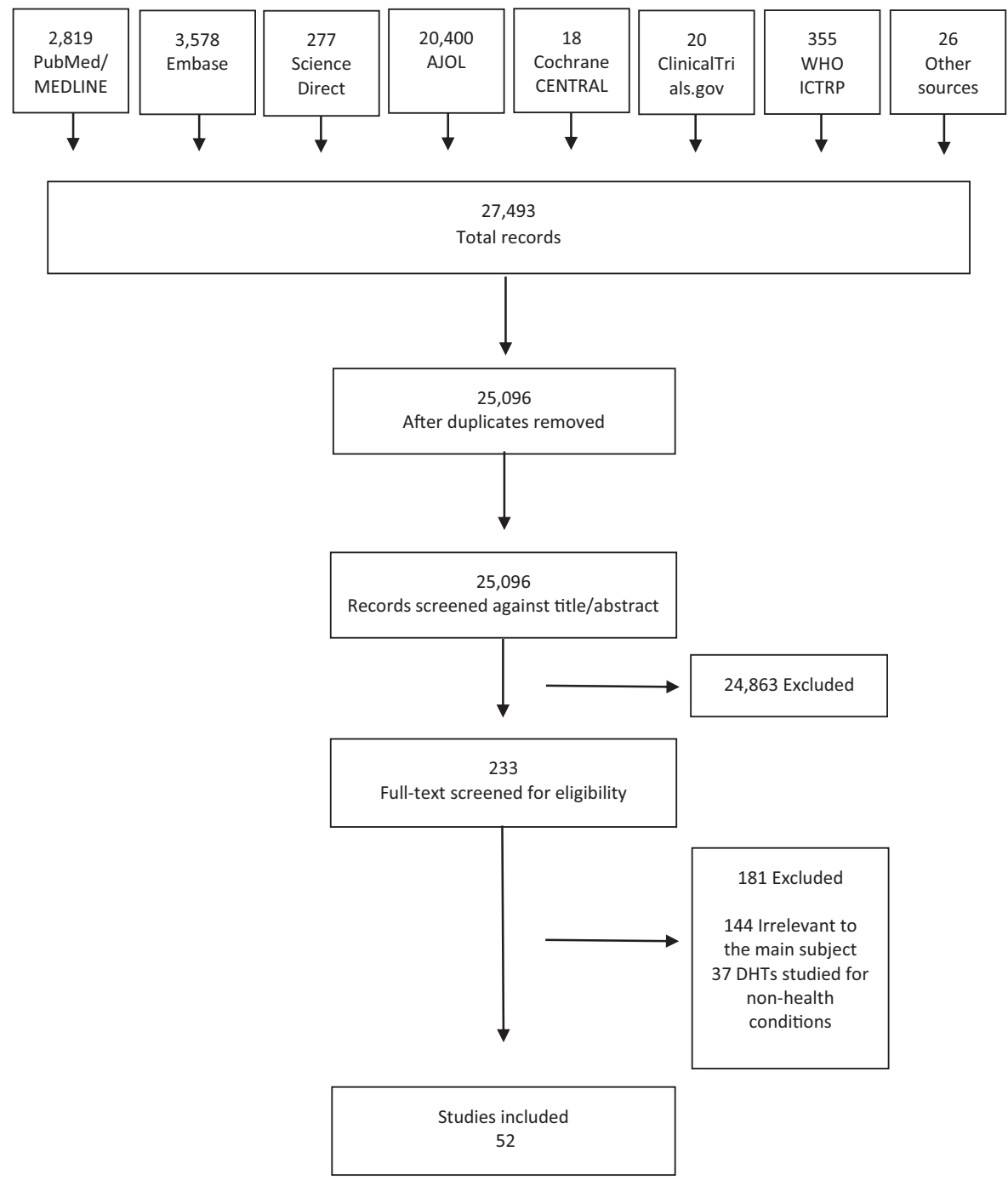

Fig. 1 PRISMA flow diagram of the study. PRISMA (preferred reporting items for systematic reviews and meta-analyses) flow diagram of included studies. 
Fifty-two (52) studies were identified that met the inclusion criteria. Figure 1 summarizes the PRISMA flowchart of the study.

Participants: The 52 included studies had a total of 596,128 study participants. The health conditions studied were maternal and child health, including antenatal care, postnatal care, infant feeding, contraceptives, and delivery $(n=15)$, infectious diseases including TB, HIV, malaria, lymphatic filariasis, and onchocerciasis $(n=14)$, non-communicable diseases including diabetes and cancer $(n=3)$, dermatitis $(n=1)$, surgery $(n=4)$, and the remaining 15 had general health conditions.

Interventions: The included studies were involved six digital health domains: 29 on mobile health (mHealth) (574,649 participants); 13 on EHR (4534 participants); four on telemedicine (465 participants); two on Cloud usage (2382 participants); three on information and communication technology (ICT, 681 participants), and one on $\mathrm{Al}$ (13,417 participants). Our search did not find articles relevant to wearable devices, software as a medical device, computing sciences in big data, cybersecurity, wireless medical devices, and robotics.

Outcomes: The primary outcome variables were feasibility ( $n=12)$, usability $(n=9)$, willingness or readiness to use ( $n=10)$, effectiveness $(n=10)$, quality improvement $(n=7)$, and knowledge or attitude about the DHT $(n=4)$. Some of the studies employed two or more of these outcomes.

Study type: The study designs were cross-sectional study $(n=41), \mathrm{RCT}$ or non-randomized experimental (6), and cohort (5).

Study setting: All the studies were conducted in Ethiopia. Some of the studies were multi-country with a significant number of participants included from Ethiopia.

\section{mHealth}

There were 29 publications identified in the $\mathrm{mHealth}$ field ${ }^{48-76}$ involving a total of 574,649 study participants: 402,542 patients, 171,295 healthy individuals, and 812 healthcare professionals. The most common citations were on the potential use of mHealth for maternal, child, and reproductive health, covering 52\% (15/29) of the citations, followed by infectious diseases, $48 \%$ (14/29). The publications were mainly cross-sectional, $69 \%$ (20/29), followed by RCT, $17 \%$ (5/29). The outcomes of interest were effectiveness ( $n=$ $9)$, usability $(n=5)$, feasibility $(n=6)$, quality $(n=4)$, willingness $(n=4)$, and knowledge $(n=1)$. Table 1 summarizes the characteristics of the included mHealth studies.

The studies had emerging insights into the potential use of mHealth to transform healthcare and improve access to services by addressing financial, social, or geographic factors, though the findings were not consistent. In a study that compared the costeffectiveness of facility-based, stand-alone, and mobile-based HIV voluntary counseling and testing, the results revealed a costeffective and improved VCT service with the use of mobile phones when compared with the two arms ${ }^{48}$. A study that assessed the potential of telephone calls to identify and follow-up post-surgery infections and total complications reported the telephone calls were feasible and valuable ${ }^{50}$. A similar study that assessed the potential of cell phones to follow-up patients after short-term surgical missions found the tool as cost-effective and reliable ${ }^{52}$. The use of SMS-based education was found to be a good option for improving the knowledge and awareness of parents regarding infant feeding ${ }^{49}$.

Several studies assessed the potential of using mHealth for family planning and maternal and child health services. One study reported that a high proportion of pregnant women in an antenatal care clinic had a mobile phone and were willing to receive an SMS text message-based mHealth intervention, though two-third of the participants lacked smartphones to upload some application software ${ }^{62}$. Locally customized mHealth applications during antenatal care significantly improved delivery and postnatal care service utilization through positively influencing the behavior of health workers and their clients ${ }^{71}$. Mobile phone reminders were effective in terms of enhancing adherence to postnatal care appointments, with the potential improving postnatal appointment adherence ${ }^{69}$. On the contrary, a retrospective analysis of demographic and health survey data reported that mobile phone ownership or receiving family planning information via SMS had no significant effect on improving contraceptive uptake ${ }^{51}$.

A study that assessed the potential of mHealth on antiretroviral (ART) services for people with HIV reported that the willingness of patients to receive SMS in support of medication reminders was not consistent, with $49 \%$ unwilling to receive the reminders ${ }^{73}$. Age, educational status, and previous experience using the internet had a significant association with their willingness ${ }^{73}$. A cross-sectional study of patients with diabetes showed that a high proportion of the patients had access to mobile phones and were willing to use them for medication reminders ${ }^{58}$.

Ten of the 29 included studies on mHealth had "healthcare professionals" as their target participants. Several of these studies revealed that mHealth had the potential to improve skills and competency of healthcare providers toward safe birth ${ }^{57}$, data quality and flow ${ }^{63}$, patient follow-up ${ }^{63}$, community-based tuberculosis and maternal health service delivery ${ }^{64}$, quality and costeffective reporting of lymphatic filariasis, and podoconiosis data ${ }^{65}$, and timely and complete reporting of maternal health data $^{67,70,74,76}$. Some healthcare providers were able to appropriately use mHealth technologies for patient assessment and routine data collection with minimal training and supervision. However, there were major preconditions needed for the healthcare providers to effectively apply such technologies, including in-service application training ${ }^{54}$, strong connectivity and electric power supply, especially in rural areas of the country ${ }^{63}$, and uninterrupted mobile network airtime ${ }^{74}$. One study reported on genomics data ${ }^{61}$. A psychiatric genomics consortium exchanged data between research groups for genome-wide association studies on neurogenetics of schizophrenia, with tens of thousands of patients and controls included. The consortium developed and used an mHealth application, MiGene Family History App (MFHA), to assist clinicians with the collection and analysis of patient genetic data over six months and assessed its feasibility through a survey of 47 clinicians. The results showed the potential expansion of medical genetics services into low and middle-income countries (LMICS) and the feasibility and benefit of the MFHA for the services ${ }^{61}$.

\section{Electronic health records}

Of the final set of 52 studies, 13 were on $\mathrm{EHR}^{77-89}$, involving 4534 study participants: 4232 health professionals, 250 patients, and 52 healthy individuals. The studies used health information technology, health management information system (HMIS), tablet-based electronic data capture (EDC), electronic information source (EIS), health smart card (HSC), and Android-based data collection system as their EHR of interest. Five studies focus on infectious diseases, one on non-communicable diseases (diabetes), and the remaining seven had a broader health system scope. The studies were all cross-sectional, 92\% (12/13), except for one RCT. The outcomes of interest were willingness $(n=5)$, usability $(n=3)$, quality $(n=3)$, and feasibility $(n=2)$. Table 2 summarizes the characteristics of the included EHR studies.

According to the studies reviewed, EHRs have the potential to exchange real-time patient-related data for better clinical decision-making and to capture and share electronic health information efficiently. However, the studies also reported potential gaps and drawbacks associated with EHRs. A study that compared EHR with paper-based records for ART data reported a higher incomplete data with the use of EHR for various reasons including difficulties implementing EHR in high patient load 


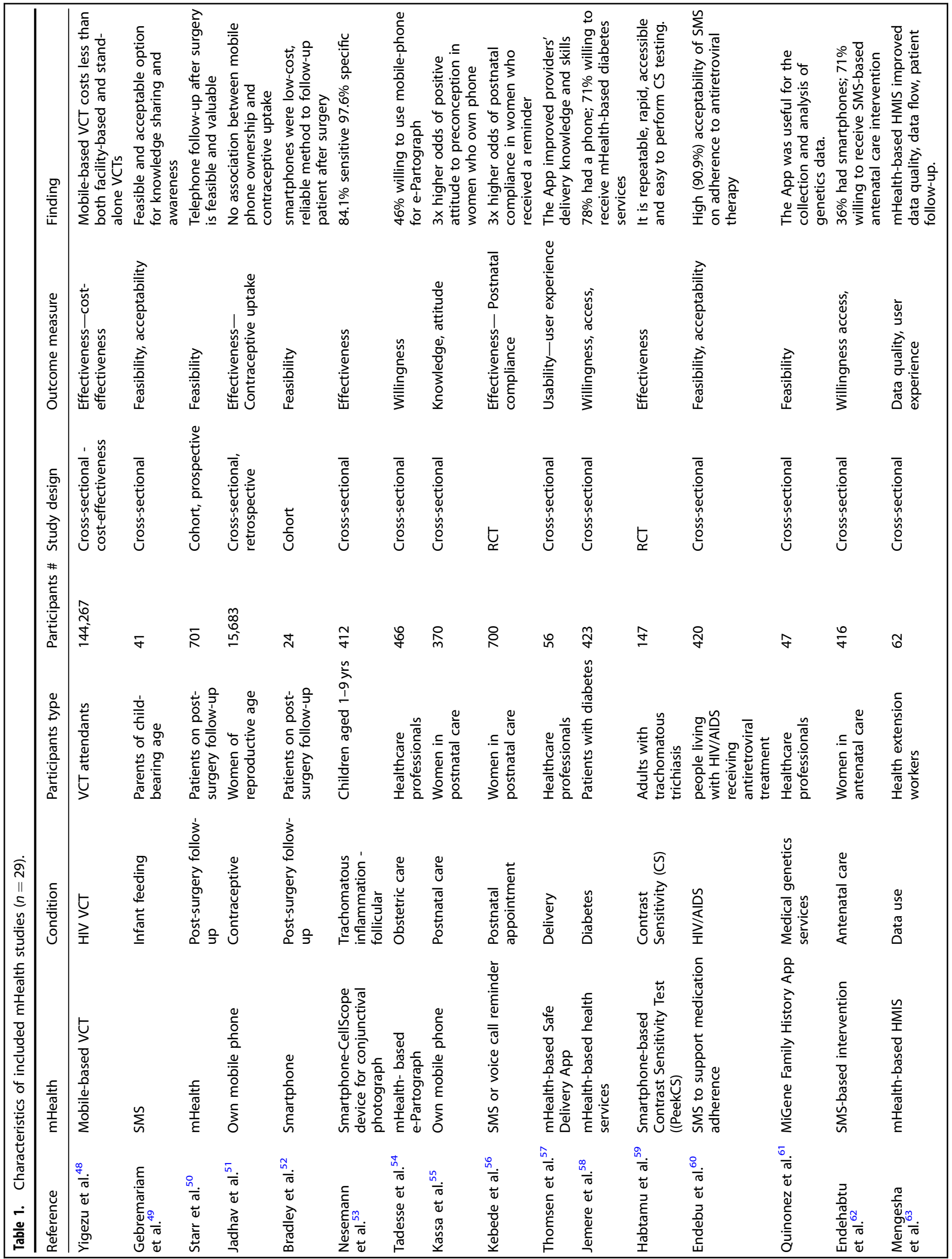




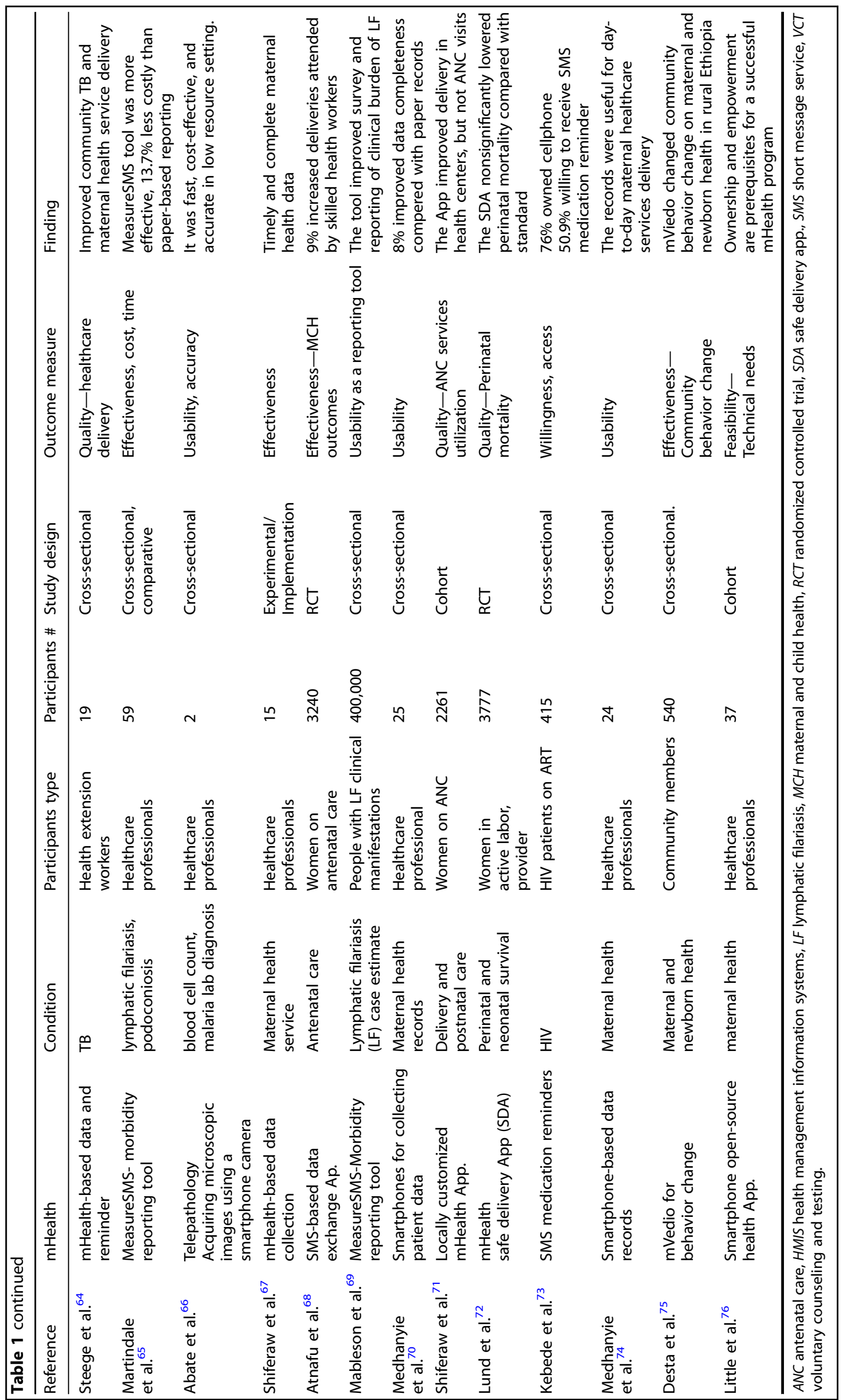




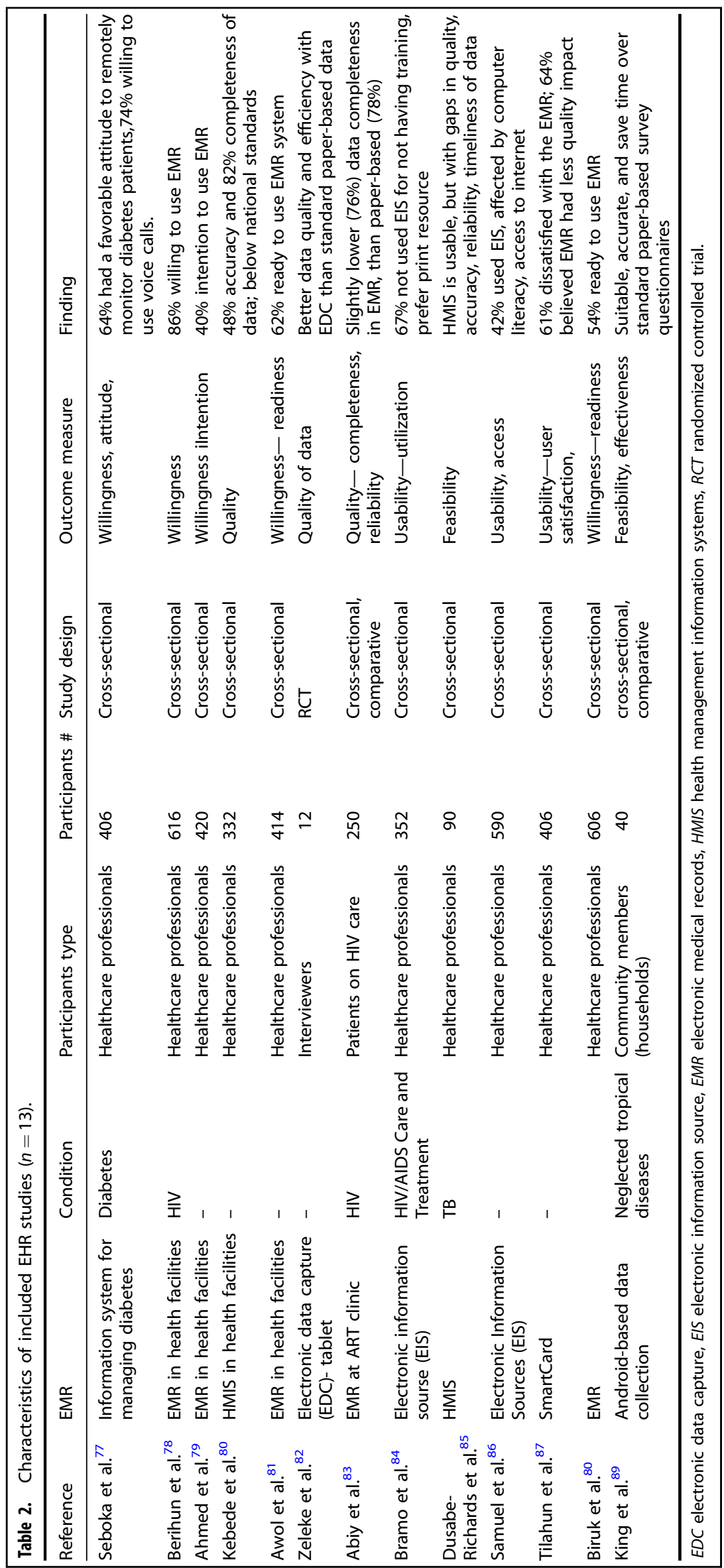


conditions and the frequent need for capturing dual electronic and paper-based data for individual patients ${ }^{83}$.

Studies that assessed the current HMIS practice in healthcare facilities reported several gaps in the accuracy, completeness, and timeliness of data for reasons including poor support from facility management, lack of accountability for data errors, poor supportive supervision, and absence of a dedicated Information Management unit responsible for EHR functions ${ }^{80,85}$.

A significant number of healthcare professionals were either not ready ${ }^{81,84,88}$ or not willing ${ }^{78,87}$ to use EHR. Some healthcare professionals still preferred paper-based records to EHR in their daily work ${ }^{82,85,87-89}$ while other preferred EHR ${ }^{82}$. Lack of access to EHR training, computer skills, and performance expectancy were the major barriers to their willingness or intention to use $\mathrm{EHR}^{77-81,83,84,87,88}$.

\section{Telemedicine}

Of the 52 studies considered, four reported on telemedicine, involving 465 participants who were healthcare professionals ${ }^{90-93}$. The studies investigated what level of knowledge and attitude healthcare professionals have toward telemedicine ${ }^{90}$, why healthcare providers resist using telemedicine ${ }^{91}$, and how telemedicine and teledermatology systems can be improved in a given program $^{92,93}$. All were cross-sectional and their outcomes of interest were knowledge and attitude, willingness, usability, and feasibility (Table 3).

The studies highlighted that telemedicine has the potential improving healthcare; however, healthcare providers had less knowledge and information about it. One study reported that of the 312 healthcare professionals included, 62\% lacked good knowledge and $36 \%$ lacked a good attitude about telemedicine ${ }^{90}$. Healthcare professionals resisted the use of telemedicine in their clinical practices mainly due to their perceived threat and controllability, with reduced autonomy, anxiety, and costs indirectly aggravating the resistance ${ }^{91}$. Telemedicine implementation in Ethiopia is influenced by technological dynamics, e-government preparedness, enabling policy environment, multistakeholder engagement, and capacity building ${ }^{93}$.

\section{Cloud-based applications}

Two studies reported on Cloud-based interventions, involving 2382 participants: 1748 surgical cases ${ }^{94}$ and 634 healthy women on cervical cancer screening ${ }^{95}$. The aims of the studies were on the feasibility of a multicentre Cloud-based peri-operative registry for surgical care ${ }^{94}$ and the feasibility of a cloud-based electronic data system for human papillomavirus (HPV) cervical cancer screening ${ }^{95}$. Table 4 summarizes the characteristics of the included Cloud studies.

A Network for Peri-operative Critical care (N4PCC) developed and evaluated a multicentre Cloud-based peri-operative registry in Ethiopia ${ }^{94}$. The authors reported on 1748 consecutive surgical cases for key performance indicators including compliance with the World Health Organization's Surgical Safety Checklist, adverse events during anesthesia, and surgical site infections. With these, the authors reported a successful multicentre digital surgical registry that can enable the measurement of key performance indicators for surgery and evaluation of peri-operative outcomes $^{95}$.

One study conducted home-based human papillomavirus (HPV) self-sampling assisted by a Cloud-based electronic data system. The study used an electronic app-based data system with an offline mode function for tablet computers, based on a Cloud solution. The app-based data system showed robust technical functionality, stability, comfort, data accuracy, and ease-of-use by health workers, with no data loss observed. The offline data collection, uploading, and synchronization system were safe and error-free ${ }^{95}$. 


\section{Artificial intelligence}

One study evaluated the precision of $\mathrm{Al}$ in differentiating between target and implanted intraocular lens (IOL) power in cataract outreach campaigns in Ethiopia ${ }^{96}$. The study applied machine learning $(\mathrm{ML})$ to optimize the $\mathrm{IOL}$ inventory and minimize avoidable refractive error in patients from the cataract campaigns ( $n=13,417)$. The result indicated good precision, with the ML optimized the implanted intraocular lens inventory and minimized avoidable refractive error (Table 5).

\section{Information communication technology (ICT)}

Three studies reported on ICT, involving a total of 681 participants: 551 healthcare professionals ${ }^{97,98}$ and 130 patients $^{99}$ (Table 6). One study ${ }^{97}$ assessed the digital competency of healthcare providers in seven public health centers and found low-level competency, with factors such as sex, educational status, profession type, monthly income, and years of experience were statistically significant predictors. The second study ${ }^{98}$ assessed health professional's behavioral intention to adopt eHealth systems and revealed that among the different eHealth constructs, healthcare professionals' attitude toward eHealth had the strongest effect on the intention to use eHealth systems. The third study evaluated the accuracy of a live-streamed video conference over a third-generation (3G) network for consultation of ultrasound scans from a remote location for a variety of pediatric indications and found the method accurate and feasible ${ }^{99}$.

\section{DISCUSSION}

We conducted a systematic review of the available literature to provide strong evidence on the potential impact of DHTs on clinical and public health practices in the context of a resource-constrained sub-Saharan African country, Ethiopia. The review identified 52 studies across different areas of DHTs, including mHealth, EMR, telemedicine, cloud-based technology, ICT, and Al. Of the 52 included studies, emerging DHTs had a very small share at $13 \%$ : Al $2 \%$, Cloud-based technology $4 \%$, and telemedicine $8 \%$, while the major $81 \%$ share was for mHealth (56\%), EHR (25\%), and ICT $6 \%$ ). This analysis demonstrated that only $10 \%(5 / 52)$ of the studies were tested in RCTs to provide robust and more credible evidence of the potential of the DHTs. Digital health solutions have substantial benefits and considerable potential to transform the healthcare system and societal wellbeing in Ethiopia. However, their adoption and implementation in full capacity face challenges in terms of infrastructure, training, access to better devices such as smartphones, and some hesitancies from patients and providers. Such challenges have been reported in studies from other African countries including Uganda ${ }^{100,101}$, Kenya ${ }^{102-105}$, and Tanzania ${ }^{106,107}$. A meta-analysis was not conducted due to the heterogeneous nature of the compiled studies.

The mHealth solutions identified in this systematic review mainly aimed to improve maternal and child healthcare and services. This review found that mHealth interventions, either a phone call or SMS, were feasible and acceptable for improving contraceptive uptake, maternal healthcare, and tuberculosis medication adherence among the Ethiopian population. The evidence also showed the potential utility of mHealth for HIV counseling and testing, outpatient follow-up, post-surgery followup, child-immunization follow-up, pregnant women antenatal and postnatal follow-ups, and in improving knowledge and awareness of parents regarding infant feeding, while its potential for contraceptive uptake was not significant. The included studies revealed that a significant number of study participants owned mobile phones and were willing to participate in mHealth-related clinical or public health interventions. However, the type of mobile phone that the patients own may not be smartphones to support an upload of needed software. Such challenges were also reported 


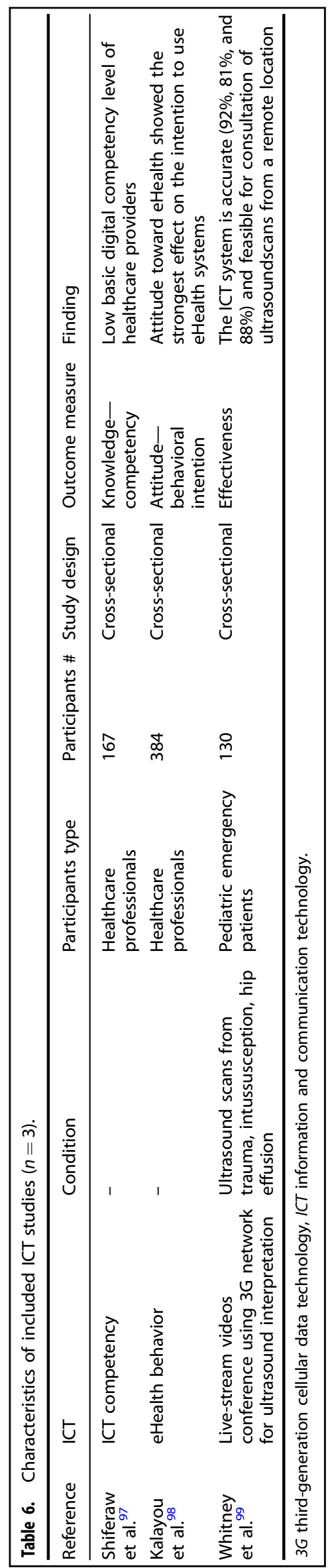


elsewhere in Kenya ${ }^{108-110}$ and Tanzania ${ }^{111}$ where there exist similar socioeconomic disparities in mobile phone ownership in support of the implementation of mHealth.

Our analysis indicates that patients with HIV may resist having their ART adherence information followed up using electronic medication reminders for fear of potential disclosure of their HIV status. The finding was consistent with a recent study in Tanzania that fear for potential involuntary disclosure of HIV status significantly affects mHealth interventions in such patients ${ }^{112}$. The development of the next generation of mHealth in such developing countries requires a broad understanding of the local social contexts that may affect the successes of DHTs ${ }^{113}$. Currently, various innovative DHTs are emerging to address the multifaceted problems associated with tuberculosis diagnosis, care and prevention. However, available data are limited for stronger conclusions of their effectiveness in various countries and settings, including Ethiopia. An RCT is currently ongoing in Ethiopia ${ }^{114}$ to bridge this gap. An initial synthesis of the evidence on DHTs is thus essential to better understand the overall digital health ecosystem in the country and successfully implement DHT-enabled healthcare and research programs.

Our analysis indicates that the use of cloud computing could help resource-constrained countries like Ethiopia to acquire advanced data storage, servers, and databases without investing in new IT infrastructure, though we have identified only two studies that are less likely to support its potential. There have been controversies on the potential benefits of the Cloud and the issues surrounding legal and regulatory implications ${ }^{115}$. For countries like Ethiopia that have not yet established a standardized legal cybersecurity framework, strategy, and governance at the national level ${ }^{116}$, adopting appropriate laws and building technical capacity would reassure the partnership and uptake of Cloud services.

Telemedicine was an emerging technology in the Ethiopian healthcare system which had its drawbacks on successful implementations, despite positive energy that healthcare providers to step up. Building capacity of the healthcare providers before full-scale implementation could bring real benefits out of telemedicine. In the COVID-19 pandemic that restricted physical contacts $^{117-119}$, telemedicine revealed significant contributions in Ethiopia by connecting patients with their healthcare providers to discuss and follow-up their disease conditions ${ }^{120}$.

In recent years, capacities for research, development, and trade on DHTs are rising sharply, while more work is needed to delineate the mechanisms of how the gains could be shared out with resource-constrained countries and global digital health strategy met. Our analysis demonstrated the feasibility and potential demands of DHTs, with the greatest opportunities in emerging health technology markets in Ethiopia.

\section{CONCLUSION}

DHTs hold much promise tackling major clinical and public health backlogs and strengthening health systems in Ethiopia. Although they are a relatively recent phenomenon in Ethiopia, their potential harnessing clinical and public health practices are highly visible. More RCTs are needed on emerging DHTs including artificial intelligence, big data, cloud, cybersecurity, telemedicine, and wearable devices to provide robust evidence of their potential use in such settings and to materialize the Global Digital Health Strategy.

\section{METHODS}

\section{Study design}

This study was based on a systematic review of scientific literature utilizing the Preferred Reporting Items for Systematic Review and Meta-Analysis Protocols (PRISMA-P) 2015 guidelines for the design and reporting of the results. The protocol was registered with PROSPERO (CRD42021240645).

To broaden the scope of DHTs in our review, we combined the latest descriptions of digital health given by the $\mathrm{WHO}^{41}$ and the $\mathrm{U}$. S. Drug and Food Administration (FDA) ${ }^{121}$. With this, the following technologies were included in the review: mobile health, telehealth, electronic health records (EHR), telemedicine, health information technology, wearable devices, software as a medical device, artificial intelligence, machine learning, genomics, computing sciences in big data, cybersecurity, wireless medical devices, and medical device interoperability.

\section{Search strategy}

We searched the PubMed-MEDLINE, Embase, ScienceDirect, African Journals Online, Cochrane Central Registry of Controlled Trials, ClinicalTrials.gov, and the WHO International Clinical Trials Registry Platform databases from inception to the latest 02 February 2021 for studies of any design and in any setting in Ethiopia that investigated the potential of DHTs in clinical or public health practices in Ethiopia. We performed manual searches of the WHO website, Google search engine, and reference lists of included studies, and contacted authors of original studies to retrieve extra possible articles or additional data. See the search strategy in the Supplementary information (Supplementary Note 1).

We tailored search strategies to each database and used controlled medical subject headings (MeSHs) and search filters where available, or Boolean search methods and free-text terms, referring to Ethiopia and Digital OR Mobile OR Smartphone OR "Cell phone" OR Techno* OR "short message service" OR SMS OR

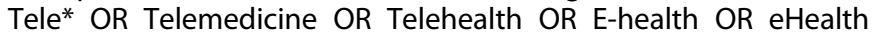
OR Remote OR Electro* OR Comput* OR cloud OR Software OR Application OR Robotics OR Blockchain OR "Artificial intelligence" OR genomics OR "big data" OR cybersecurity OR wireless.

\section{Eligibility}

Studies were included if they met the following inclusion criteria:

Participants: Eligible participants could be patients, healthcare professionals, data collectors, or healthy individuals in Ethiopia, either women or men, and without age restrictions. Thus, the search was not restricted to participants except that they should reside in Ethiopia.

Interventions: All DHTs that were included in our definition. Studies that investigated digital technologies for non-health conditions were excluded.

Comparisons: Studies with a comparison condition were not required as a criterion. Thus, studies with or without a comparator were eligible.

Outcome: Studies assessing the potential efficacy, effectiveness, feasibility, usability, acceptability, or any related outcomes were included in the review without specific restrictions.

Study design: All available study designs were included. We excluded reviews, commentaries, editorials, and proceedings as these are non-empirical publications.

\section{Study selection}

Two independent authors examined the title and abstract of all screened publications. From the title and abstract of all publications identified by the database search, those that were duplicated or did not meet the inclusion criteria were excluded. The full texts of the remaining publications were further reviewed. Disagreements were resolved by consensus and, if persisted, were arbitrated through discussion with a third author.

\section{Data extraction}

The identified data were listed, and information was provided on the type and details of the type of DHT under investigation, the 
disease condition studied, the type of participants, the number of participants, the study design employed, outcome measures, major findings, the surname of the first author, and year of publication.

\section{Data management and analysis}

The publications were grouped in exhaustive tables based on the type of DHT investigated. A qualitative content analysis of all documents and articles was performed. Each article was summarized, and the data were reported descriptively. Less emphasis was placed on the assessment of the quality of the included literature as that was not the major objective of this review.

This analysis was designed to inform our ongoing DHTenabled randomized controlled trial (RCT) on tuberculosis in Ethiopia (ClinicalTrials.gov, ID: NCT04216420). The trial aims to evaluate the effectiveness of a digital health technology-enabled self-administered therapy over standard directly observed therapy on adherence to TB medication and treatment outcomes in Ethiopia ${ }^{114}$.

\section{Operational definitions}

Mobile health (mHealth): The use of mobile phone device's core utility of voice or short messaging service (SMS) as well as more complex functionalities to improve health outcomes and healthcare services.

Electronic health records (EHRs): are patient-centered electronic records that provide immediate and secure information to authorized users.

Telemedicine: The practice of medicine at a distance which involves an interaction between a healthcare provider and a patient when the two are separated by distance.

Cloud: The practice of storing and computing health data remotely over the internet, which is managed by external service providers.

Artificial intelligence (Al): The simulation of human intelligence in a digital computer that is programmed to think or perform health tasks like humans.

Information and communications technology (ICT): technologies that provide access to health information through telecommunications.

\section{DATA AVAILABILITY}

All the data included in this study are available within the paper and its Supplementary Information files.

Received: 4 March 2021; Accepted: 24 June 2021;

Published online: 17 August 2021

\section{REFERENCES}

1. Shapiro, A. et al. A novel digital approach to describe real world outcomes among patients with constipation. NPJ Digit. Med. 4, 27 (2021).

2. Daly, A. C., Prendergast, M. E., Hughes, A. J. \& Burdick, J. A. Bioprinting for the biologist. Cell 184, 18-32 (2021).

3. Zhang, Z. et al. EPHA7 mutation as a predictive biomarker for immune checkpoint inhibitors in multiple cancers. BMC Med. 19, 26 (2021).

4. Tschandl, P. et al. Human-computer collaboration for skin cancer recognition. Nat. Med. 26, 1229-1234 (2020).

5. Røttingen, J. A. \& Farrar, J. Targeted health innovation for global health. BMJ 366, 15601 (2019).

6. Schulman, K. A. \& Richman, B. D. Toward an effective innovation agenda. N. Engl. J. Med. 380, 900-901 (2019).

7. Klonoff, D. C., King, F. \& Kerr, D. New opportunities for digital health to thrive. J. Diabetes Sci. Technol. 13, 159-163 (2019).
8. Navarro-Torné, A., Hanrahan, F., Kerstiëns, B., Aguar, P. \& Matthiessen, L. Public health-driven research and innovation for next-generation influenza vaccines, european union. Emerg. Infect. Dis. 25, e180359 (2019).

9. Brown, E. L. et al. Smartphone-based health technologies for dementia care: opportunities, challenges, and current practices. J. Appl. Gerontol. 38, 73-91 (2019).

10. Pham, L. et al. Smartphone-based symbol-digit modalities test reliably captures brain damage in multiple sclerosis. NPJ Digit. Med. 4, 36 (2021).

11. Subudhi, $S$. et al. Comparing machine learning algorithms for predicting ICU admission and mortality in COVID-19. NPJ Digit. Med. 4, 87 (2021).

12. Kvedar, J. C. \& Fogel, A. L. mHealth advances clinical research, bit by bit. Nat. Biotechnol. 35, 337-339 (2017).

13. Seto, E., Smith, D., Jacques, M. \& Morita, P. P. Opportunities and challenges of telehealth in remote communities: case study of the Yukon telehealth system. JMIR Med. Inf. 7, e11353 (2019).

14. Dunn, P. \& Hazzard, E. Technology approaches to digital health literacy. Int. J. Cardiol. 293, 294-296 (2019).

15. Burlone, S., Moore, L. \& Johnson, W. Overcoming barriers to accessing obstetric care in underserved communities. Obstet. Gynecol. 134, 271-275 (2019).

16. Liao, Y., Thompson, C. \& Peterson, S. The future of wearable technologies and remote monitoring in health care. Am. Soc. Clin. Oncol. Educ. Book 39, 115-121 (2019).

17. Piao, C., Terrault, N. A. \& Sarkar, S. Telemedicine: an evolving field in hepatology. Hepatol. Commun. 3, 716-721 (2019).

18. Fogel, A. L. \& Kvedar, J. C. Artificial intelligence powers digital medicine. NPJ Digit. Med. 1, 5 (2018)

19. Young, A. T. et al. Stress testing reveals gaps in clinic readiness of image-based diagnostic artificial intelligence models. NPJ Digit. Med. 4, 10 (2021).

20. Randhawa, G. K. \& Jackson, M. The role of artificial intelligence in learning and professional development for healthcare professionals. Health. Manag. Forum 33, 19-24 (2020).

21. Wiljer, D. \& Hakim, Z. Developing an artificial intelligence-enabled health care practice: rewiring health care professions for better care. J. Med. Imaging Radiat. Sci. 50, S8-S14 (2019).

22. Chiu, Y. C. et al. Deep learning of pharmacogenomics resources: moving towards precision oncology. Brief. Bioinform. 21, 2066-2083 (2020).

23. Ritchie, M. D., Moore, J. H. \& Kim, J. H. Translational bioinformatics: biobanks in the precision medicine era. Pac. Symp. Biocomput. 25, 743-747 (2020).

24. Lienhardt, C. \& Nahid, P. Advances in clinical trial design for development of new TB treatments: a call for innovation. PLoS Med. 16, e1002769 (2019).

25. Lehoux, P. et al. What health system challenges should responsible innovation in health address? Insights from an international scoping review. Int. J. Health Policy Manag. 8, 63-75 (2019).

26. Félix, I. B. et al. Development of a complex intervention to improve adherence to antidiabetic medication in older people using an anthropomorphic virtual assistant software. Front. Pharm. 10, 680 (2019).

27. Shakeri Hossein Abad, Z. et al. Digital public health surveillance: a systematic scoping review. NPJ Digit. Med. 4, 41 (2021).

28. Cornell University, INSEAD, and the World Intellectual Property Organization. Global innovation index 2019: Creating health lives - the future of medical innovation. 12th ed. https://www.wipo.int/edocs/pubdocs/en/wipo_pub_gii_2019. pdf (Ithaca, Fontainebleau, and Geneva, 2019).

29. United Nations Conference on Trade and Development (UNCTED). Selected sustainable development trends in the least developed countries-2019. https://unctad.org/en/PublicationsLibrary/aldc2019d1_en.pdf (Geneva, 2019).

30. Shahin, M. H. et al. The patient-centered future of clinical pharmacology. Clin. Pharm. Ther. 107, 72-75 (2020).

31. Barrenho, E., Miraldo, M. \& Smith, P. C. Does global drug innovation correspond to burden of disease? The neglected diseases in developed and developing countries. Health Econ. 28, 123-143 (2019).

32. Burki, T. Developing countries in the digital revolution. Lancet 391, 417 (2018)

33. Oloruntoba, S. O. \& Muchie, M. Innovation, Regional Integration, and Development in Africa: Advances in African Economic, Social and Political Development. (Springer, 2019).

34. Mackintosh, M. et al. Health-industry linkages for local health: reframing policies for African health system strengthening. Health Policy Plan 33, 602-610 (2018).

35. United Nations Conference on Trade and Development (UNCTED). Technology and innovation report-2018: Harnessing Frontier Technologies for Sustainable Development. https://unctad.org/en/PublicationsLibrary/tir2018_en.pdf (Geneve, 2018).

36. Mugabe, J. O. World Health Organization. Health innovation systems in developing countries: strategies for building scientific and technological capacities. (World Health Organization, 2005).

37. Simpkin, V. et al. Investing in health R\&D: where we are, what limits us, and how to make progress in Africa. BMJ Glob. Health 4, e001047 (2019). 
38. Nabyonga-Orem, J., Nabukalu, J. B. \& Okuonzi, S. A. Partnership with private forprofit sector for universal health coverage in sub-Saharan Africa: opportunities and caveats. BMJ Glob. Health 4, e001193 (2019).

39. Olakunde, B. O. et al. Revisiting aid dependency for HIV programs in SubSaharan Africa. Public Health 170, 57-60 (2019).

40. Taura, N. D., Bolat, E. \& Madichie, N. O. (eds). Digital Entrepreneurship in SubSaharan Africa: Challenges, Opportunities and Prospects. (Springer, 2019).

41. World Health Organization (WHO). Global Strategy on Digital Health 2020-2025. https://www.who.int/docs/default-source/documents/gs4dhdaa2a9f352b0445b afbc79ca799dce4d.pdf (WHO, Geneva, Switzerland, 2020).

42. World Health Organization Regional Office for Africa (WHO-AFRO). Health Technologies and Innovations. https://www.afro.who.int/programmes-clusters/ HTI (2019).

43. World Bank. World Bank in Ethiopia: Overview. https://www.worldbank.org/en/ country/ethiopia/overview (World Bank, 2019).

44. J. ohn Snow, Inc. (JSI). Ethiopia Launches Digital Health Innovation and Learning Center.|NEWS.https://www.jsi.com/ethiopia-launches-digital-health-innovationand-learning-center/ (August 6th, 2020).

45. Ethiotelecom. Statistics. Addis Ababa, Ethiopia, Ethiotelecom. https://www. ethiotelecom.et/ (April, 2021).

46. Ethiopian News Agency (ENA). Global Partnership for Ethiopia Group Awarded Nationwide Telecom License. Addis Ababa, Ethiopia; ENA. https://www.ena.et/ en $/ p=24642$ (22 May, 2021).

47. Ethiopia Ministry of Health. The Ethiopian Health Sector Transformation Plan II. https://www.moh.gov.et/ejcc/am/node/152 (Ethiopian Ministry of Health, 2019, Addis Ababa, Ethiopia).

48. Yigezu, A. et al. Cost-effectiveness of facility-based, stand-alone and mobilebased voluntary counseling and testing for HIV in Addis Ababa, Ethiopia. Cost. Eff. Resour. Alloc. 18, 34 (2020).

49. Gebremariam, K. T., Zelenko, O., Hadush, Z., Mulugeta, A. \& Gallegos, D. Could mobile phone text messages be used for infant feeding education in Ethiopia? A formative qualitative study. Health Inform. J. 26, 2614-2624 (2020).

50. Starr, N. et al. Value and feasibility of telephone follow-up in ethiopian surgical patients. Surg. Infect. (LarChmt.) 21, 533-539 (2020).

51. Jadhav, A. \& Weis, J. Mobile phone ownership, text messages, and contraceptive use: Is there a digital revolution in family planning? Contraception 101, 97-105 (2020).

52. Bradley, D. et al. Smartphones can be used for patient follow-up after a surgical mission treating complex head and neck disfigurement in Ethiopia: Results from a prospective pilot study. J. Plast. Reconstr. Aesthet. Surg. 74, 890-930 (2021).

53. Nesemann, J. M. et al. Comparison of smartphone photography, single-lens reflex photography, and field-grading for trachoma. Am. J. Trop. Med. Hyg. 103, 2488-2491 (2020).

54. Tadesse, Y. et al. Willingness to use mobile based e-partograph and associated factors among care providers in North Gondar Zone, Northwest Ethiopia. Online J. Public Health Inf. 11, e10 (2019).

55. Kassa, Z. Y. et al. Mobile phone based strategies for preconception education in rural Africa. Ann. Glob. Health 85, 101 (2019).

56. Kebede, A. S., Ajayi, I. O. \& Arowojolu, A. O. Effect of enhanced reminders on postnatal clinic attendance in Addis Ababa, Ethiopia: a cluster randomized controlled trial. Glob. Health Action 12, 1609297 (2019).

57. Thomsen, C. F. et al. Health workers' experiences with the safe Delivery App in West Wollega Zone, Ethiopia: a qualitative study. Reprod. Health 16, 50 (2019).

58. Jemere, A. T. et al. Access to mobile phone and willingness to receive mHealth services among patients with diabetes in Northwest Ethiopia: a cross-sectional study. BMJ Open 9, e021766 (2019).

59. Habtamu, E. et al. Development and Validation of a Smartphone-based Contrast Sensitivity Test. Transl. Vis. Sci. Technol. 8, 13 (2019).

60. Endebu, T., Deksisa, A., Dugasa, W., Mulu, E. \& Bogale, T. Acceptability and feasibility of short message service to improve ART medication adherence among people living with HIV/AIDS receiving antiretroviral treatment at Adama hospital medical college, Central Ethiopia. BMC Public Health 19, 1315 (2019).

61. Quinonez, S. C. et al. Introducing medical genetics services in Ethiopia using the MiGene Family History App. Genet. Med. 21, 451-458 (2019).

62. Endehabtu, B. et al. Mobile phone access and willingness among mothers to receive a text-based mhealth intervention to improve prenatal care in Northwest Ethiopia: cross-sectional study. JMIR Pediatr. Parent 1, e9 (2018).

63. Mengesha, W., Steege, R., Kea, A. Z., Theobald, S. \& Datiko, D. G. Can mHealth improve timeliness and quality of health data collected and used by health extension workers in rural Southern Ethiopia? J. Public Health (Oxf.) 40, ii74-ii86 (2018).

64. Steege, R. et al. 'The phone is my boss and my helper'-a gender analysis of an mHealth intervention with Health Extension Workers in Southern Ethiopia. J. Public Health (Oxf.) 40, ii16-ii31 (2018).

65. Martindale, S. et al. A comparison between paper-based and $\mathrm{m}$-Health tools for collating and reporting clinical cases of lymphatic filariasis and podoconiosis in Ethiopia. Mhealth 4, 49 (2018).
66. Abate, A., Kifle, M., Sena Okuboyejo, S. \& Mbarika, V. A mobile-based telepathology system for a low resource setting in Ethiopia. Appl. Comput. Inform. 14, 186-191 (2018)

67. Shiferaw, S., Workneh, A., Yirgu, R., Dinant, G. J. \& Spigt, M. Designing mHealth for maternity services in primary health facilities in a low-income setting - lessons from a partially successful implementation. BMC Med. Inf. Decis. Mak. 18, 96 (2018).

68. Atnafu, A., Otto, K. \& Herbst, C. H. The role of mHealth intervention on maternal and child health service delivery: findings from a randomized controlled field trial in rural Ethiopia. Mhealth 3, 39 (2017).

69. Mableson, H. E., Martindale, S., Stanton, M. C., Mackenzie, C. \& Kelly-Hope, L. A. Community-based field implementation scenarios of a short message service reporting tool for lymphatic filariasis case estimates in Africa and Asia. Mhealth 3, 28 (2017)

70. Medhanyie, A. A. et al. Quality of routine health data collected by health workers using smartphone at primary health care in Ethiopia. Int J. Med. Inf. 101, 9-14 (2017).

71. Shiferaw, S. et al. The Effects of a locally developed mhealth intervention on delivery and postnatal care utilization; a prospective controlled evaluation among health centres in Ethiopia. PLoS ONE 11, e0158600 (2016).

72. Lund, S. et al. Association between the safe delivery app and quality of care and perinatal survival in Ethiopia: a randomized clinical trial. JAMA Pediatr. 170, 765-771 (2016).

73. Kebede, M., Zeleke, A., Asemahagn, M. \& Fritz, F. Willingness to receive text message medication reminders among patients on antiretroviral treatment in North West Ethiopia: A cross-sectional study. BMC Med. Inf. Decis. Mak. 15, 65 (2015).

74. Medhanyie, A. A. et al. Health workers' experiences, barriers, preferences and motivating factors in using mHealth forms in Ethiopia. Hum. Resour. Health 13, 2 (2015).

75. Desta, B. F. Use of mobile video show for community behavior change on maternal and newborn health in rural Ethiopia. J. Midwifery Women's Health 59, S65-S72 (2014).

76. Little, A. et al. Meeting community health worker needs for maternal health care service delivery using appropriate mobile technologies in Ethiopia [published correction appears in Ethiopia. [published correction]. PLoS ONE 8, e77563 (2013).

77. Seboka, B. T., Yilma, T. M. \& Birhanu, A. Y. Factors influencing healthcare providers' attitude and willingness to use information technology in diabetes management. BMC Med Inf. Decis. Mak. 21, 24 (2021).

78. Berihun, B., Atnafu, D. D. \& Sitotaw, G. Willingness to use electronic medical record (EMR) system in healthcare facilities of Bahir Dar City, Northwest Ethiopia. Biomed. Res. Int. 2020, 3827328 (2020).

79. Ahmed, M. H. et al. Intention to use electronic medical record and its predictors among health care providers at referral hospitals, north-West Ethiopia, 2019: using unified theory of acceptance and use technology 2(UTAUT2) model. BMC Med Inf. Decis. Mak. 20, 207 (2020).

80. Kebede, M., Adeba, E. \& Chego, M. Evaluation of quality and use of health management information system in primary health care units of east Wollega zone, Oromia regional state, Ethiopia. BMC Med Inf. Decis. Mak. 20, 107 (2020).

81. Awol, S. M. et al. Health professionals' readiness and its associated factors to implement electronic medical record system in four selected primary hospitals in Ethiopia. Adv. Med Educ. Pract. 11, 147-154 (2020).

82. Zeleke, A. A. et al. Evaluation of electronic and paper-pen data capturing tools for data quality in a public health survey in a health and demographic surveillance site, Ethiopia: randomized controlled crossover health care information technology evaluation. JMIR Mhealth Uhealth 7, e10995 (2019).

83. Abiy, R. et al. A comparison of electronic medical record data to paper records in antiretroviral therapy clinic in Ethiopia: what is affecting the quality of the data? Online J. Public Health Inf. 10, e212 (2018).

84. Bramo, S. S. \& Agago, T. A. Utilization status of electronic information sources (EIS) for HIV/AIDS care and treatment in specialized teaching hospitals of Ethiopia, 2016. Ethiop. J. Health Sci. 27, 507-514 (2017).

85. Dusabe-Richards, J. N. et al. Women health extension workers: capacities, opportunities and challenges to use eHealth to strengthen equitable health systems in Southern Ethiopia. Can. J. Public Health 107, e355-e361 (2016).

86. Samuel, S., Bayissa, G., Asaminewu, S. \& Alaro, T. Electronic information sources access and use for healthcare services in governmental and non-governmental hospitals of Western Oromia, Ethiopia: a cross sectional study. Ethiop. J. Health Sci. 26, 341-350 (2016)

87. Tilahun, B. \& Fritz, F. Comprehensive evaluation of electronic medical record system use and user satisfaction at five low-resource setting hospitals in ethiopia. JMIR Med. Inf. 3, e22 (2015).

88. Biruk, S., Yilma, T., Andualem, M. \& Tilahun, B. Health professionals' readiness to implement electronic medical record system at three hospitals in Ethiopia: a cross sectional study. BMC Med. Inf. Decis. Mak. 14, 115 (2014). 
89. King, J. D. et al. A novel electronic data collection system for large-scale surveys of neglected tropical diseases. PLoS ONE 8, e74570 (2013).

90. Biruk, K. \& Abetu, E. Knowledge and attitude of health professionals toward telemedicine in resource-limited settings: a cross-sectional study in North West Ethiopia. J. Health. Eng. 2018, 2389268 (2018).

91. Xue, Y. et al. Investigating the resistance to telemedicine in Ethiopia. Int J. Med. Inf. 84, 537-547 (2015).

92. Delaigue, S., Morand, J. J., Olson, D., Wootton, R. \& Bonnardot, L. Teledermatology in low-resource settings: the MSF experience with a multilingual tele-expertise platform. Front Public Health 2, 233 (2014).

93. Shiferaw, F. \& Zolfo, M. The role of information communication technology (ICT) towards universal health coverage: the first steps of a telemedicine project in Ethiopia. Glob. Health Action 5, 1-8 (2012).

94. Network for Peri-operative Critical care (N4PCC)*. Addressing priorities for surgical research in Africa: implementation of a multicentre cloud-based perioperative registry in Ethiopia. Anaesthesia 76, 933-939 (2021).

95. Jede, F. et al. Home-based HPV self-sampling assisted by a cloud-based electronic data system: Lessons learnt from a pilot community cervical cancer screening campaign in rural Ethiopia. Papillomavirus Res. 9, 100198 (2020).

96. Brant, A. R. et al. Artificial intelligence in global ophthalmology: using machine learning to improve cataract surgery outcomes at Ethiopian outreaches. J. Cataract Refract Surg. 47, 6-10 (2020).

97. Shiferaw, K. B., Tilahun, B. C. \& Endehabtu, B. F. Healthcare providers' digital competency: a cross-sectional survey in a low-income country setting. $B M C$ Health Serv. Res. 20, 1021 (2020).

98. Kalayou, M. H., Endehabtu, B. F. \& Tilahun, B. The applicability of the modified technology acceptance model (TAM) on the sustainable adoption of eHealth systems in resource-limited settings. J. Multidiscip. Healthc. 13, 1827-1837 (2020).

99. Whitney, R. E., Riera, A. \& Chen, L. Accuracy of remote interpretation of pediatric emergency ultrasound over third generation networks across continents. Pediatr. Emerg. Care 36, e340-e342 (2020).

100. Meyer, A. J. et al. Implementing mHealth interventions in a resource-constrained setting: case study from Uganda. JMIR Mhealth Uhealth 8, e19552 (2020).

101. Mugambe, R. K. et al. Impact of mhealth messages and environmental cues on hand hygiene practice among healthcare workers in the greater Kampala metropolitan area, Uganda: study protocol for a cluster randomized trial. BMC Health Serv. Res. 21, 88 (2021).

102. Aw, M. et al. Critical appraisal of a mHealth-assisted community-based cardiovascular disease risk screening program in rural Kenya: an operational research study. Pathog. Glob. Health 114, 379-387 (2020).

103. Karlyn, A. et al. Testing mHealth solutions at the last mile: insights from a study of technology-assisted community health referrals in rural Kenya. Mhealth 6, 43 (2020).

104. Ngongo, B. P., Ochola, P., Ndegwa, J. \& Katuse, P. The technological, organizational and environmental determinants of adoption of mobile health applications ( $\mathrm{m}$-health) by hospitals in Kenya. PLoS ONE 14, e0225167 (2019).

105. Harrington, E. K. et al. An mHealth SMS intervention on postpartum contraceptive use among women and couples in Kenya: a randomized controlled trial. Am. J. Public Health 109, 934-941 (2019).

106. Klingberg, A., Sawe, H. R., Hammar, U., Wallis, L. A. \& Hasselberg, M. m-Health for burn injury consultations in a low-resource setting: an acceptability study among health care providers. Telemed. J. E Health 26, 395-405 (2020).

107. Thomas, D. S., Daly, K., Nyanza, E. C., Ngallaba, S. E. \& Bull, S. Health worker acceptability of an mHealth platform to facilitate the prevention of mother-tochild transmission of HIV in Tanzania. Digit Health 6, 2055207620905409 (2020).

108. Lee, S., Begley, C. E., Morgan, R., Chan, W. \& Kim, S. Y. Addition of mHealth (mobile health) for family planning support in Kenya: disparities in access to mobile phones and associations with contraceptive knowledge and use. Int Health 11, 463-471 (2019).

109. Kazi, A. M. et al. Assessing mobile phone access and perceptions for textingbased mHealth interventions among expectant mothers and child caregivers in remote regions of Northern Kenya: a survey-based descriptive study. JMIR Public Health Surveill. 3, e5 (2017).

110. Johnson, D. et al. A randomized controlled trial of the impact of a family planning mHealth service on knowledge and use of contraception. Contraception 95, 90-97 (2017).

111. Thobias, J. \& Kiwanuka, A. Design and implementation of an m-health data model for improving health information access for reproductive and child health services in low resource settings using a participatory action research approach. BMC Med. Inf. Decis. Mak. 18, 45 (2018).

112. Ngowi, K. M. et al. Technical and psychosocial challenges of mHealth usage for antiretroviral therapy adherence among people living with hiv in a resourcelimited setting: case series. JMIR Form. Res 4, e14649 (2020).
113. Alaiad, A., Alsharo, M. \& Alnsour, Y. The determinants of M-Health adoption in developing countries: an empirical investigation. Appl Clin. Inf. 10, 820-840 (2019).

114. Manyazewal, T. et al. Electronic pillbox-enabled self-administered therapy versus standard directly observed therapy for tuberculosis medication adherence and treatment outcomes in Ethiopia (SELFTB): protocol for a multicenter randomized controlled trial. Trials 21, 383 (2020).

115. Webster, P. Patient data in the cloud. Lancet Digital Health 1, E391-E392 (2019).

116. Adane, K. The current status of cyber security in Ethiopia. IUP J. Inf. Technol. 16, 7-19 (2020).

117. Manyazewal, T., Woldeamanuel, Y., Blumberg, H. M., Fekadu, A. \& Marconi, V. C. The fight to end tuberculosis must not be forgotten in the COVID-19 outbreak. Nat. Med. 26, 811-812 (2020).

118. Seifu Estifanos, A. et al. 'I exist because of we': shielding as a communal ethic of maintaining social bonds during the COVID-19 response in Ethiopia. BMJ Glob. Health 5, e003204 (2020).

119. Mohammed, H. et al. Containment of COVID-19 in Ethiopia and implications for tuberculosis care and research. Infect. Dis. Poverty 9, 131 (2020).

120. Wondimu, W. \& Girma, B. Challenges and silver linings of COVID-19 in Ethiopia -short review. J. Multidiscip. Health. 13, 917-922 (2020).

121. U.S Food and Drug Administration (FDA). What is Digital Health? https://www. fda.gov/medical-devices/digital-health-center-excellence/what-digital-health (MD, USA, FDA, 2020).

\section{ACKNOWLEDGEMENTS}

The work was supported by the Fogarty International Center and National Institute of Allergy and Infectious Diseases of the US National Institutes of Health (D43TW009127) and the Emory Center for AIDS Research (P30 Al050409). The content is solely the responsibility of the authors and does not necessarily represent the official views of the National Institutes of Health or the Emory Center for AIDS Research.

\section{AUTHOR CONTRIBUTIONS}

Study conception, acquisition and synthesis of data, and first draft: T.M. Acquisition, analysis, and interpretation of data: V.C.M. and Y.W. interpretation of data and resource acquisition: H.M.B. and A.F. All authors reviewed and approved the final version for publication.

\section{COMPETING INTERESTS}

The authors declare no competing interests.

\section{ADDITIONAL INFORMATION}

Supplementary information The online version contains supplementary material available at https://doi.org/10.1038/s41746-021-00487-4.

Correspondence and requests for materials should be addressed to T.M.

Reprints and permission information is available at http://www.nature.com/ reprints

Publisher's note Springer Nature remains neutral with regard to jurisdictional claims in published maps and institutional affiliations.

\begin{abstract}
Open Access This article is licensed under a Creative Commons Attribution 4.0 International License, which permits use, sharing, adaptation, distribution and reproduction in any medium or format, as long as you give appropriate credit to the original author(s) and the source, provide a link to the Creative Commons license, and indicate if changes were made. The images or other third party material in this article are included in the article's Creative Commons license, unless indicated otherwise in a credit line to the material. If material is not included in the article's Creative Commons license and your intended use is not permitted by statutory regulation or exceeds the permitted use, you will need to obtain permission directly from the copyright holder. To view a copy of this license, visit http://creativecommons. org/licenses/by/4.0/.
\end{abstract}

(c) The Author(s) 2021 